\title{
The ecological effects of selective decontamination of the digestive tract (SDD) on antimicrobial resistance: a 21-year longitudinal single-centre study
}

Sophie Buitinck ${ }^{1,2}$, Rogier Jansen ${ }^{3}$, Saskia Rijkenberg ${ }^{1}$, Jos P. J. Wester ${ }^{1}$, Rob J. Bosman ${ }^{1}$, Nardo J. M. van der Meer ${ }^{2,4}$ and Peter H. J. van der Voort ${ }^{1,2^{*}}$ (D)

\begin{abstract}
Background: The long-term ecological effects on the emergence of antimicrobial resistance at the ICU level during selective decontamination of the digestive tract (SDD) are unknown. We determined the incidence of newly acquired antimicrobial resistance of aerobic gram-negative potentially pathogenic bacteria (AGNB) during SDD.

Methods: In a single-centre observational cohort study over a 21-year period, all consecutive patients, treated with or without SDD, admitted to the ICU were included. The antibiotic regime was unchanged over the study period. Incidence rates for ICU-acquired AGNB's resistance for third-generation cephalosporins, colistin/polymyxin B, tobramycin/gentamicin or ciprofloxacin were calculated per year. Changes over time were tested by negative binomial regression in a generalized linear model.

Results: Eighty-six percent of 14,015 patients were treated with SDD. Most cultures were taken from the digestive tract (41.9\%) and sputum (21.1\%). A total of 20,593 isolates of AGNB were identified. The two most often found bacteria were Escherichia coli $(N=6409)$ and Pseudomonas $(N=5269)$. The incidence rate per 1000 patient-day for ICU-acquired resistance to cephalosporins was 2.03, for polymyxin B/colistin 0.51 , for tobramycin 2.59 and for ciprofloxacin 2.2. The incidence rates for ICU-acquired resistant microbes per year ranged from 0 to 4.94 per 1000 patient-days, and no significant time-trend in incidence rates were found for any of the antimicrobials. The background prevalence rates of resistant strains measured on admission for cephalosporins, polymyxin B/colistin and ciprofloxacin rose over time with $7.9 \%, 3.5 \%$ and $8.0 \%$ respectively.

Conclusions: During more than 21-year SDD, the incidence rates of resistant microbes at the ICU level did not significantly increase over time but the background resistance rates increased. An overall ecological effect of prolonged application of SDD by counting resistant microorganisms in the ICU was not shown in a country with relatively low rates of resistant microorganisms.
\end{abstract}

Keywords: Selective digestive tract decontamination, SDD, Resistance, Ecological effect, Critically ill

\footnotetext{
* Correspondence: phjvdvoort@chello.nl

'Department of Intensive Care, OLVG Hospital, Oosterpark 9, 1091 AC

Amsterdam, The Netherlands

${ }^{2}$ TIAS School for Business and Society, Warandelaan 2, 5037 AB Tilburg, The

Netherlands

Full list of author information is available at the end of the article
}

(c) The Author(s). 2019 Open Access This article is distributed under the terms of the Creative Commons Attribution 4.0 International License (http://creativecommons.org/licenses/by/4.0/), which permits unrestricted use, distribution, and reproduction in any medium, provided you give appropriate credit to the original author(s) and the source, provide a link to the Creative Commons license, and indicate if changes were made. The Creative Commons Public Domain Dedication waiver (http://creativecommons.org/publicdomain/zero/1.0/) applies to the data made available in this article, unless otherwise stated. 


\section{Background}

Selective decontamination of the digestive tract (SDD) is effective in preventing intensive care unit (ICU)-acquired pneumonia, sepsis and subsequent mortality [1-4]. This is due to the elimination of potentially pathogenic microbes from the digestive tract using topical antibiotics [5]. As a consequence, secondary colonization and subsequent endogenous infection are prevented [5]. This routine use of antimicrobials raises concerns for antimicrobial resistance, and these concerns are the most important reason to oppose the use of SDD [6]. Some reports, however, show that the elimination of pathogens from the bowel might even reduce the emergence of resistant strains [7-10]. Two meta-analyses on the effect of selective oral decontamination (SOD) and SDD on the development of antimicrobial resistance showed no relation between the use of SDD or SOD and the emergence of antimicrobial resistance $[11,12]$. Most included studies were randomized controlled trials that focussed on individual patients treated with SDD but they seldomly included the patients in the ICU without SDD. As a consequence, at the ICU level, in contrast to the individual patient level, the effect on microbial resistance could not be determined with these studies. Moreover, these studies generally reported a relatively short inclusion period.

The aim of the present study is to determine the incidence of antimicrobial resistance in aerobic gram-negative potentially pathogenic microorganisms (AGNB) to the components of SDD and other frequently used antibiotics at ICU level over a 21-year period with an unchanged antibiotic policy.

\section{Methods}

\section{Study design, setting and patients}

This study has a single-centre longitudinal observational cohort design. The patient data are registered in a local database since 1997 and retrospectively extracted for this analysis. The study is conducted in a Dutch 20-bed adult mixed medical, surgical and cardiac surgery tertiary intensive care unit. All consecutive patients admitted to the ICU between January 1997 and July 2017 were included, and clinical data were extracted. All available cultures and patient data were used. Patients without cultures taken during ICU treatment could not be counted in the culture data but are included in the other analyses. Data on baseline characteristics of all included patients were prospectively recorded in the ICU database and extracted for this analysis.

The local medical ethical review board (ACWO OLVG) approved the study and waived informed consent due to its retrospective design in accordance with Dutch and European legislation (study no. WO 13.063).

\section{Cultures}

Data on all cultures taken during ICU stay of the included patients were over the years registered in the hospital database and retrospectively retrieved from this database. Both surveillance cultures (throat, rectum, tracheal aspirate) and cultures of organ sites and blood taken during ICU stay were included for analysis. Routinely, cultures from the throat and rectum were taken twice a week and tracheal aspirate three times a week for surveillance in patients treated with SDD. For nonintubated patients, the tracheal aspirate was substituted for sputum when available. All culture samples taken in the context of surveillance were plated on an unselective blood agar and four specific agars selecting for grampositive bacteria, gram-negative bacteria, yeast and vancomycin-resistant enterococci. Gram-negatives were cultured on McConkey agar; Staphylococcus aureus was detected using both unselective blood agar and Columbia aztreonam polymyxin agar. For yeasts, Sabouraud maltose agar was used. Cultured gram-negative bacteria were tested for antimicrobial susceptibility using agar disk diffusion during the entire study period. Cutoff values for resistance were set following the guidelines of the 'Clinical and Laboratory Standards Institute' (CLSI) [13] until July 2011 and 'the European Committee on Antimicrobial Susceptibility Testing' (EUCAST) from July 2011 until 2017 [14]. Cutoff values for resistance for colistin were performed according to CLSI ranges, for the whole study period.

\section{Antimicrobial treatment 1997-2017}

Patients were treated with SDD when the expected ICU stay was more than $24 \mathrm{~h}$, with or without mechanical ventilation. This relatively liberal use of SDD is based on the concept that acquisition of potential pathogenic microorganisms starts in the first day of ICU stay $[15,16]$. SDD has been consistently used since 1986 following the same protocol. The SDD regimen consists of four times daily Orabase ${ }^{\oplus}$ oral paste with $2 \%$ polymyxin $\mathrm{B}$, amphotericin $\mathrm{B}$ and tobramycin. In addition, $10 \mathrm{ml}$ of a suspension containing $500 \mathrm{mg}$ amphotericin $\mathrm{B}, 100 \mathrm{mg}$ polymyxin $\mathrm{B}$ and $80 \mathrm{mg}$ tobramycin is administered four times daily in the gastric tube or swallowed in patients without a gastric tube. Cefotaxime is administered four times daily $1 \mathrm{~g}$ i.v. for 4 days in all SDD-treated patients but is administered longer in case of active infection. For active infection, the coverage of gram-negatives can be extended with ciprofloxacin i.v. or tobramycin i.v. when needed. In case of peritonitis, metronidazole is added as well. Other i.v. antimicrobials can be given based on previous culture results. Penicillins are carefully avoided whenever possible due to their negative effects on the normal aerobic and anaerobic intestinal flora which could lead to a loss of the protective effect to invading 
pathogens (colonization resistance). Methicillin-resistant staphylococci (MRSA) colonization and infection were consistently treated with vancomycin i.v. and $2 \%$ vancomycin added in the oral paste and gastric suspension. When a combined polymyxin and tobramycin resistance was present in AGNB from surveillance cultures, then co-trimoxazole $2 \%$ was added in the oral paste and twice daily $960 \mathrm{mg}$ in the enteral suspension when appropriate and until decontamination was obtained [15].

\section{Data analysis and statistics}

Cultures with aerobic gram-negative potentially pathogenic bacteria (AGNB) were selected. Among others, these AGNB are Acinetobacter spp., Citrobacter spp., Escheria coli, Enterobacter spp., Klebsiella spp., Morganella spp., Proteus spp., Pseudomonas aeruginosa, and Serratia marcescens [16]. To obtain the resistant strains for analysis, we selected in every patient the AGNB with antimicrobial resistance for tobramycin/gentamicin, polymyxin $\mathrm{B} /$ colistin, cephalosporins or ciprofloxacin and counted the first culture with this particular resistance. The strains were defined as resistant when they were tested intermediate or resistant in vitro. Strains that were resistant on admission were marked as not ICU-acquired and were excluded for the calculation of the incidence rates but they were analyzed separately to calculate the background resistance rates on admission. Resistant pathogens were considered ICU-acquired if these pathogens were absent in the first 48 to $72 \mathrm{~h}$ after admission but present in subsequent cultures. In case a patient enters the ICU with resistant bacteria and subsequently acquires other resistant bacteria, they are counted in both groups. Because of variability in time of admission and time that the culture was taken, the cultures taken on day three are considered within 48 to $72 \mathrm{~h}$ after admission. As a consequence, all resistant pathogens from cultures taken on day of admission (day one), day two or day three were considered resistant on admission and not ICU acquired. This is consistent with the definition of ICU-acquired infection in the ECDC HAIICU protocol for healthcare associated infections in intensive care units' [17].

\section{Prevalence rate and incidence rate}

The incidence rates for ICU-acquired resistance were calculated for each year for second- or third-generation cephalosporins (including cefotaxime, ceftriaxone, cefuroxim and ceftazidim), polymyxin $\mathrm{B} /$ colistin, tobramycin/ gentamicin and ciprofloxacin separately. Microorganisms that were intrinsically resistant to one of the antimicrobial agents were excluded for the analysis of that specific agent. For third-generation cephalosporins, we excluded Serratia marcescens, Acinetobacter spp., Citrobacter freundii, Enterobacter spp. and Morganella morgannii, and for polymyxinB/colistin, Serratia marcescens, Proteus spp. and Morganella spp. were excluded for this calculation. Incidence rates were calculated instead of cumulative incidence because of the dynamic population [18]. The incidence rate was defined as:

[Number of ICU-acquired resistant PPMs/sum of the person-time of the population at risk] $\times 1000$.

Population at risk was defined as all patients admitted to the ICU during the study period without resistant strains on admission. Patients who were treated less than $48 \mathrm{~h}$ in the ICU were excluded from the denominator as they could not have acquired new (multi)resistant strains in this short period. The person-time, calculated in patient-days per year, per patient at risk is defined as the number of days between ICU admission and discharge from the ICU. The number of days was calculated without correcting for the time of admission or discharge. The day of admission is 'day one' and so forth. In case of acquisition of a resistant potentially pathogenic microorganism (PPM), person-time was defined as the number of patientdays between ICU admission and acquisition of this resistant PPM. The total number of patient-days for patients at risk was calculated for every antimicrobial agent separately. Incidence rates were calculated for patients with an ICU length of stay more than $48 \mathrm{~h}$ and for all patients (Additional files 1, 2, 3 and 4), for medical and surgical patients (Additional files 1, 2, 3 and 4).

\section{Statistical analyses}

Continuous data are shown as mean and standard deviation (SD) or median and interquartile range (IQR) depending on the distribution. Changes in incidence over time were evaluated by negative binomial regression in a generalized linear model, as the assumption for Poisson distribution that mean and variance are equal was not met (overdispersion). The dependent variable is the incidence rate per 1000 patient-days of ICU-acquired resistant strains for a given antimicrobial agent over the years of measurement. The Omnibus test was used to test for significance. It compares the model including the variable 'year' to the intercept-only model to see if there is a significant time-trend. A two-sided $p$ value below 0.05 was considered statistically significant.

To determine background resistance rates, the prevalence of resistance on admission in AGNB was calculated for each year separately. The prevalence was calculated by dividing the number of resistant strains on admission by the number of admissions in that particular year. All data were analyzed using Statistical Package of Social Science (SPSS 24.0 Chicago, IL, USA).

\section{Results}

\section{Patients and cultures}

Between January 1, 1997, and July 29, 2017, 15,051 patients were admitted to the ICU. These patients had 17,511 
admissions with cultures taken during ICU stay. Baseline characteristics are shown in Table 1. In the second column, all patients with an ICU length of stay less than $48 \mathrm{~h}$ are excluded as they could not acquire new colonization with resistant strains in this short period. Over time, the case mix did not change substantially with around half of the population having cardiovascular disease and the other mixed diseases including sepsis and abdominal surgery. The distribution of sample sites is also shown in Table 1. The majority of the cultures were surveillance cultures

Table 1 Baseline characteristics

All patients

Patients and cultures baseline characteristics

Number of admissions between 1997 and 2017

Age in years, mean (SD)

Males, N (\%)/females N (\%)

Length of ICU stay in days, median (IQR)

APACHE II score

Patient category

Admission type

Treated with SDD\#

ICU mortality, N (\%)

Cultures

Distribution of specimen
Median (IQR)

APACHE $\|<20, N(\%)$

APACHE $\|>=20, N(\%)$

Missing, N (\%)

Cardiothoracic surgery, N (\%)

Internal medicine, $N(\%)$

Surgery, N (\%)

Cardiology, N (\%)

Pulmonology, $N(\%)$

Neurology, $N(\%)$

Other, $N(\%)$

Missing, $N(\%)$

Medical*, N (\%)

Elective surgery, $N(\%)$

Scheduled surgery, N (\%)

Urgent surgery, $N(\%)$

Emergency surgery, N (\%)

Missing, $N(\%)$

Yes, $N(\%)$

No, $N(\%)$

Missing, $N(\%)$

Digestive tract cultures, N (\%)

Sputum cultures, N (\%)

Skin and wound cultures

Urine cultures

Blood cultures

Abdominal cavity cultures

Other

Unknown

Total number of cultures

More than $48 \mathrm{~h} \mathrm{ICU}$ stay

17,511

$65.3(14.5)$

$11,093(63.4 \%) / 6414(36.6 \%)$

$3.0(4.0)$

$20.0(11.0)$

8281 (47.3\%)

$8320(47.5 \%)$

$910(5.6 \%)$

6109 (34.9\%)

$2434(13.9 \%)$

$3361(19.2 \%)$

2468 (14.1\%)

$1529(8.7 \%)$

$527(3.0 \%)$

1009 (5.8\%)

$74(0.4 \%)$

9335 (53.3\%)

$3964(22.6 \%)$

$1264(7.2 \%)$

$1553(8.9 \%)$

$624(3.6 \%)$

$771(4.4 \%)$

$12,054(86.0 \%)$

$1043(7.4 \%)$

$919(6.6 \%)$

$2459(14.0 \%)$

68,057 (41.9\%)

$37,827(23.3 \%)$

7308 (4.5\%)

$8212(5.1 \%)$

27,884 (17.2\%)

1125 (0.7\%)

11,236 (6.9\%)

$586(0.4 \%)$

162,235
9588

66.0 (13.6)

6077 (63.4\%)/3508 (36.3\%)

$6.0(6.0)$

$22.0(11.0)$

3428 (35.8\%)

$5767(60.1 \%)$

$393(4.1 \%)$

3021 (31.5\%)

1666 (17.4\%)

1751 (18.2\%)

1540 (16.1\%)

1047 (10.9\%)

$299(3.1 \%)$

264 (2.8\%)

$3(0.03 \%)$

5787 (60.4\%)

$1696(17.7 \%)$

532 (5.5\%)

825 (8.6\%)

$358(3.7 \%)$

390 (4.1\%)

7141 (93.5\%)

$83(1.1 \%)$

416 (5.4\%)

$1537(16.0 \%)$

Continuous data are shown as mean and standard deviation (SD) in case of normal distribution

* Medical patients are all patients without previous surgery preceding ICU admission

\#Data available since 2002 
from the throat or rectum (41.9\%) or cultures retrieved from tracheal aspirate (23.3\%).

Figure 1 shows the number of cultures and number of resistant strains. A total of 20,593 isolates of AGNB were identified. These included Proteus spp. $(N=2857)$, Klebsiella spp. $(N=1634)$, Morganella spp. $(N=759)$, Enterobacter spp. $(N=1571)$, Citrobacter spp. $(N=529)$, Serratia marcescens $(N=1081)$, Pseudomonas spp. $(N=5269)$, Acinetobacter spp. $(N=485)$ and $E$. coli $(N=6409)$.

For the second- or third-generation cephalosporins, the number of isolates that were tested for resistance increased from $78.4 \%$ in 1997 to $99.2 \%$ in 2017. A higher test rate leads to a better detection of resistant microorganisms. Susceptibility for ciprofloxacin was tested for $78.4 \%$ in 1997 and $99.2 \%$ in 2017 . In $65.8 \%$ of isolates, information on susceptibility to polymyxin $\mathrm{B} /$ colistin was available. The number of tested isolates for polymyxin B/colistin rose from $22.7 \%$ in 1997 to $99.0 \%$ in 2017. Information on susceptibility for aminoglycosides was available in $92.9 \%$ of all isolates. This number varied between 78.8 and $99.2 \%$ over the years. More information on susceptibility testing per year for the different antimicrobial agents is shown in Additional files 1, 2,3 and 4 .

The incidence rate per 1000 patient-days per year is shown in Fig. 2. These data reflect the population of ICU patients with a length of stay more than $48 \mathrm{~h}$. In Additional files 1, 2, 3 and 4, the data including the patients with a shorter length of stay are shown. The average incidence rate for ICU-acquired resistance to third-generation cephalosporins was 2.03 per 1000 patientdays (range $0.55 / 1000$ patient-days in 2001 to $4.3 / 1000$ patient-days in 2012), 0.51 per 1000 patient-days for polymyxin B/colistin (range $0.00 / 1000$ patient-days in 1997 to 2.12/1000 patient-days in 2010), 2.59 per 1000 patient-days for tobramycin (range $1.09 / 1000$ in 2004 to $4.08 / 1000$ patient-days in 2012) and 2.20 per 1000 patient-days for ciprofloxacin (range 0.26/1000 patient-days in 1998 to 4.94 per 1000 patient-days in 2009. The results of the negative binomial regression analysis are listed in Table 2.

The incidence rates for medical and surgical patients have been calculated separately. Additional files 3 and 4 Medical and Surgical show the incidence rates over time for these two groups. Basically, it is shown that in both

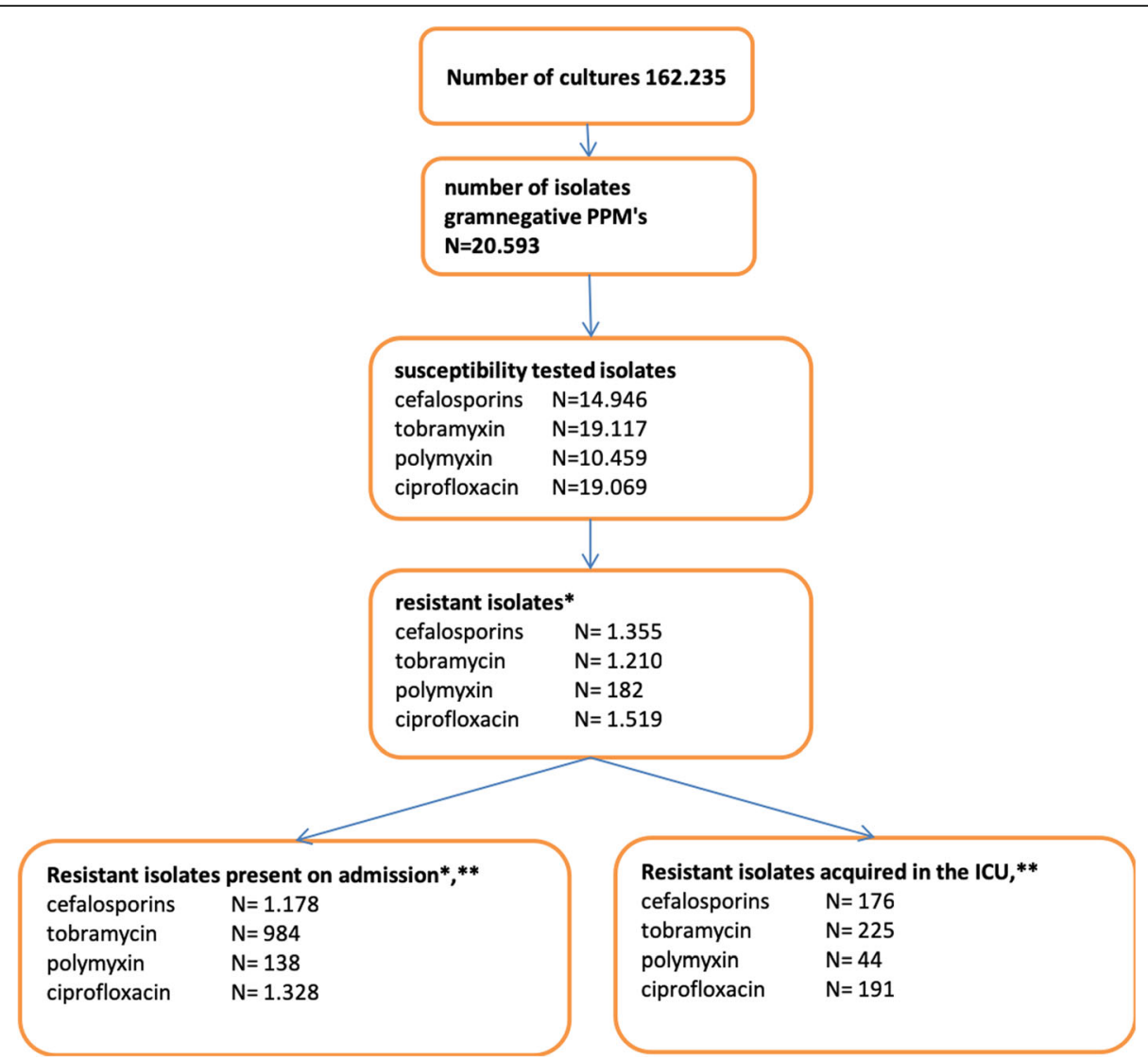

Fig. 1 Flowchart of cultures. *Only first resistant isolate mentioned in flowchart. **First culture with antimicrobial resistance taken within $72 \mathrm{~h}$ after admission 


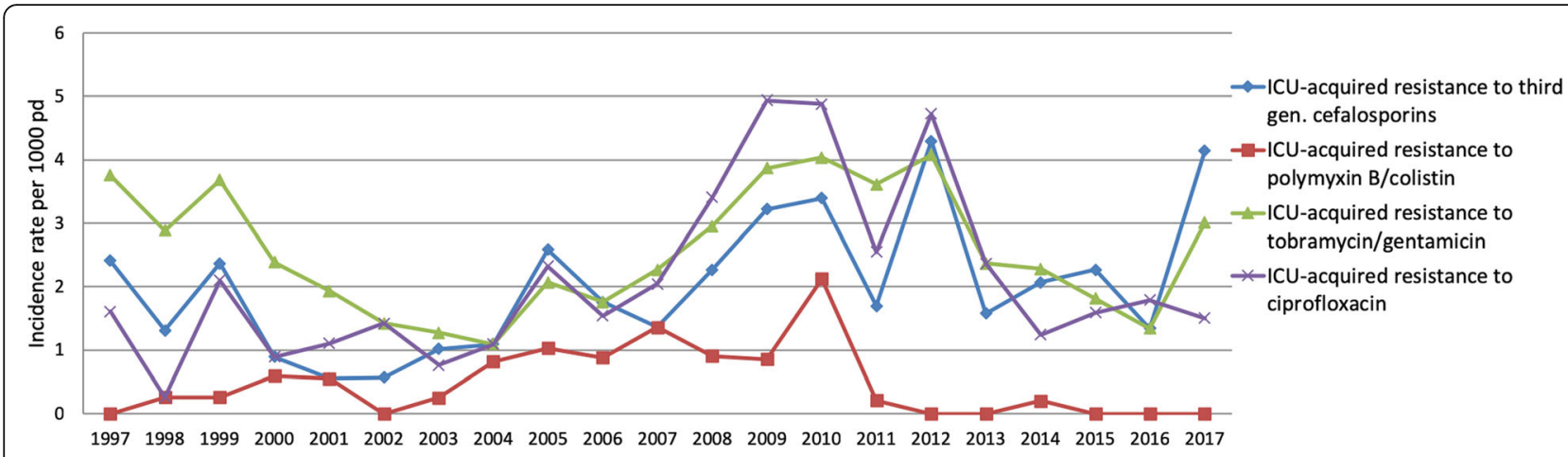

Fig. 2 Incidence rates per 1000 patient-days for acquired resistance

groups the incidence rates are low with some variability over the years.

The prevalence rates of resistant aerobic gram-negative PPMs on admission per year are presented in Fig. 3, and this represents the 'background' prevalence. Overall prevalence rate of resistance to cephalosporins was 6.7 per 100 ICU admissions. This was 0.8/100 admissions for polymyxin $\mathrm{B} /$ colistin, 5.6/100 admissions for tobramycin and 7.6/100 admissions for ciprofloxacin. Figure 3 shows an increase in background resistance for cephalosporins, ciprofloxacin and tobramycin but for polymyxin $\mathrm{B} /$ colistin this trend is not seen.

\section{Discussion}

This study shows that some fluctuation in the incidence of newly acquired resistant microorganisms over 21 years of SDD exists but a significant or relevant increase in resistance over the study period was not found despite the fact that the number of patients with one or more resistant strains on admission was steadily increasing. The long-term ecological effects of SDD on ICU level concerning antimicrobial resistance of aerobic gramnegative PPMs to the components of SDD appear to be limited. The incidence rates for ICU-acquired bacteria resistant for third-generation cephalosporins, tobramycin/ gentamicin, polymyxin $\mathrm{B} /$ colistin and ciprofloxacin appeared to be very low in this study, between 0 and 6 per 1000 patient-days for each antimicrobial agent. We analyzed the data excluding and including patients with a length of stay less than $48 \mathrm{~h}$ and for medical and surgical patients separately. Basically, all analyses showed the same result. These findings are consistent with most of the previous studies on the emergence of antimicrobial resistance during SDD use [10-12, 19-23]. Halaby et al., in contrast, showed an increased prevalence (the incidence was not reported) of colistin-resistant microorganisms which probably was due to failure of hygienic measures in that ICU [24]. In addition, Oostdijk et al. reported a trend to an increased incidence of aminoglycosides [25]. Moreover, several studies showed a decrease in resistance after implementation of SDD [10, 19-21].

This study is the first to report uninterrupted use of SDD over a time period of 21 years. As SDD started in 1985 in this ICU, in fact, more than 30 years of uninterrupted use did not lead to emergence of resistance. We can only report 21 years as the electronic registration of culture results started in 1994. Studies on the long-term effects of the emergence of resistant microorganism and studies at the ICU level of SDD are scarce [12]. The second strength of this study is that patients without SDD treatment have been included as well. As a result, a more comprehensive overview of the effects of SDD can be obtained at the unit level. Some of these effects might have stayed unrecognized when in a RCT design, only patients that are included in the study would be evaluated leaving others, potentially carrying resistant strains, unnoticed. Other reservoirs in the ICU than patients, such as sinks or equipment, were not checked for the presence of resistant microorganisms in this study. In the context

Table 2 Incidence of acquired antimicrobial resistance over time

\begin{tabular}{lllll}
\hline Antimicrobial agent & All patients & LOS $>48 \mathrm{~h}$ & Surgical & Medical \\
\hline Third generation cephalosporins & $0.063(p=0.998)$ & $0.071(p=0.79)$ & $0.14(p=0.71)$ & $0.009(p=0.92)$ \\
Tobramycin/gentamicin & $0.39(p=0.999)$ & $0.09(p=0.76)$ & $0.070(p=0.79)$ & $0.10(p=0.75)$ \\
Ciprofloxacin & $0.88(p=0.985)$ & $0.04(p=0.84)$ & $0.034(p=0.85)$ & $0.30(p=0.58)$ \\
Polymyxin B/Colistin & $0.07(p=0.899)$ & $0.26(p=0.61)$ & $0.54(p=0.46)$ & $0.46(p=0.50)$ \\
\hline
\end{tabular}

Data are shown as likelihood ratio chi-square test (Omnibus test) to compare the fitted model (model including year) against the intercept-only model with $p$ value

LOS length of stay in the ICU 


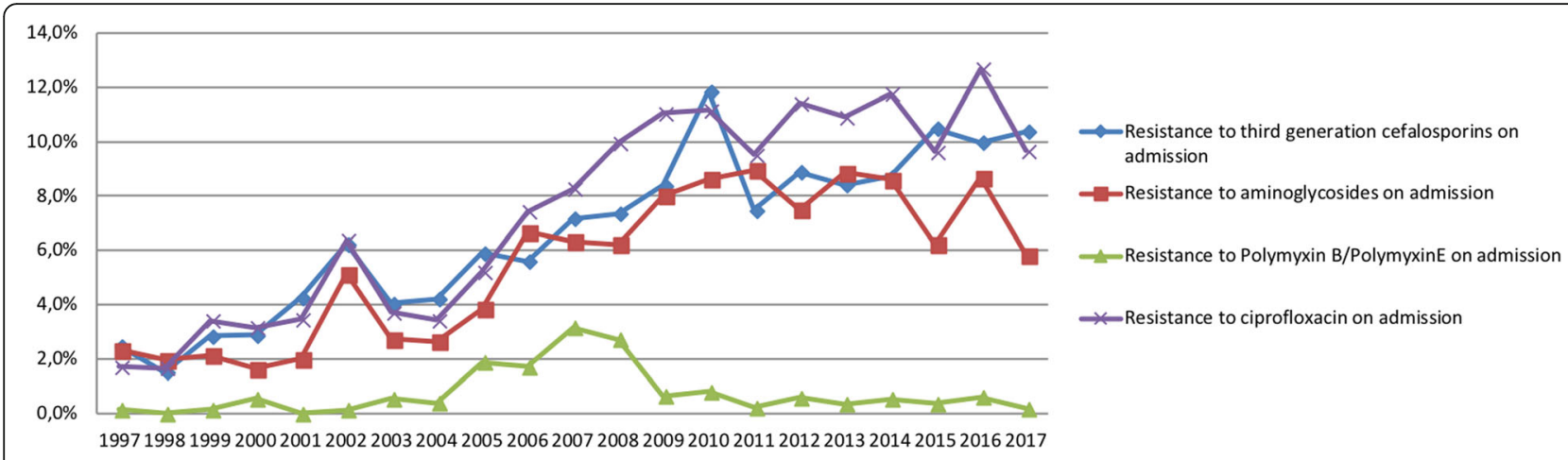

Fig. 3 Admission prevalence rates for resistant antimicrobials per year

of SDD, these reservoirs should be dealt with by hygienic measures, one of the pillars of the SDD regime.

Little changes have been made in the antibiotic policy of our ICU in the past 20 years. In particular, SDD has been used continuously, consistently and unchanged in composition during the whole 21-year study period. The exposure to the antimicrobials is therefore long enough to unravel ecological trends.

This study also has limitations. First, the longitudinal cohort analysis of this study has its methodological flaws. It cannot compare groups with or without SDD but that was not the primary aim of this study. The primary aim was to study the ecological effects on unit level for which a randomized study is less appropriate. As causal effects are difficult to determine, we can only conclude that long-term SDD use was not associated with increasing incidence of resistant strains. In addition, we did not take rectal and throat cultures from patients who were not treated with SDD which leaves us unknown about their colonization. It is unknown whether these patients introduce resistant microorganism in the ICU. Also, they might take acquired multiresistant microorganisms with them out of the ICU but the exposure (colonization pressure) appears to be low. Second, because of its mono-centred design, the extrapolation to other ICUs is difficult to make. More ICUs with the same length of SDD use were not available, which limited the analysis to a single centre. Third, in this study, we limited our resistance rates to second/third-generation cephalosporins, tobramycin/gentamicin, polymyxin $\mathrm{B} /$ colistin and ciprofloxacin in aerobic gram-negative PPMs. We did not study other antimicrobials or gram-positive microorganisms as previous studies did not find an emergence of resistant gram-positive bacteria like enterococci [26, 27].

Fourth, the guidelines for susceptibility testing have been changed several times between 1997 and 2017, which could lead to differences in classification at different time periods. In our centre, the guidelines have been changed from CLSI to EUCAST guidelines in 2011. As EUCAST is known to recommend lower resistance
MIC breakpoints, it can hypothetically lead to higher resistance rates. However, our results did not show this. This is confirmed by several studies that have been published on the effect of changing guidelines on resistance rates [28, 29]. When comparing CLSI 2009 guidelines and EUCAST 2010 guidelines, the effects of changing guidelines were small and would have led to higher instead of lower resistance rates $[28,29]$.

Fifth, detailed information on a patient level concerning the use of antibiotics in our ICU was not available for most of the study period. Also, the time window between admission and the detection of newly acquired antimicrobial resistance is unknown but would have been informative in relation to the length of stay. Sixth, we have studied this cohort in a country with a relatively low prevalence of resistant bacteria. However, we have also shown that the increasing background prevalence did not influence the incidence of newly acquired resistant strains in the ICU. This stresses the importance of hygienic measures as one of the pillars of SDD treatment [16]. In Fig. 2, a temporary increase in ICU-acquired resistance between 2007 and 2012 was seen. The subsequent decrease might be related to a policy change resulting in more patients in preventive isolation on admission, which might have reduced cross contamination from newly admitted patients to others. It may also indicate that transmission is a more important mechanism than new formation or selection of resistant strains.

We have included both patients with and without mechanical ventilation, which is not in all ICUs and studies routine. Over the years, around $85 \%$ of our population is mechanically ventilated during their stay in the ICU but we do not have exact data available for this study. It is unknown whether this 'liberal' SDD policy relates to the incidence rates that we report here. In addition, the median length of stay of all patients is 3.0 days, which is relatively short for newly acquired resistant microorganisms. Therefore, we analyzed the patients with a length of stay of more than $48 \mathrm{~h}$ who appeared to have a median length of stay of 6.0 days (Table 1 ). 
This study is performed in a setting with a low baseline prevalence of resistant microorganisms. A rise in background rates of resistant microorganisms is present, also in the Netherlands. Despite this rise (Fig. 3), watchful application of SDD and adaptation of the substances that are used when necessary did not lead to an increase in the incidence of resistant microorganisms. In a recent study, Wittekamp et al. showed no effect of SDD on bacteraemia in a setting of high prevalence of resistant microorganisms. The limitation of that study is that they did not adapt the composition of the SDD substances to the specific patterns of resistant microorganisms as we did in our unit [30].

\section{Conclusions}

This study shows that long-term use of SDD can be safe in relation to the emergence of resistant microbes as our study found that between 1997 and 2017, the incidence of ICU-acquired antimicrobial resistance to second/thirdgeneration cephalosporins, tobramycin/gentamicin, polymyxin $\mathrm{B} /$ colistin and ciprofloxacin in AGNB was low and did not increase over time with uninterrupted use of SDD. This is seen despite increased background resistance rates on admission due to an overall increase of antimicrobial resistance in the hospital population in a country with on average low rates of resistant microorganisms.

\section{Additional files}

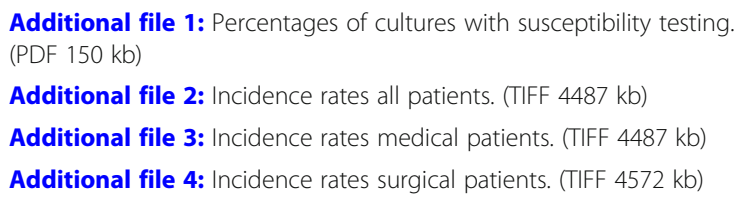

\section{Abbreviations}

AGNB: Aerobic gram-negative potentially pathogenic bacteria; CLSI: Clinical and Laboratory Standards Institute; ECDC : European Centre for Disease Prevention and Control; EUCAST: European Committee on Antimicrobial Susceptibility Testing; HAllCU: Healthcare-associated infections in intensive care units; ICU: Intensive care unit; IQR: Interquartile range; MEC: Medical Ethical review board; MRSA: Methicillin-resistant Staphylococcus aureus; PPM: Potentially pathogenic microorganism; RCT: Randomized controlled trial; SD: Standard deviation; SDD: Selective digestive tract decontamination SOD: Selective oral decontamination; SPSS: Statistical Package for the Social Science

\section{Acknowledgements}

We would like to thank C. J. van der Mark for the extensive data extraction from the microbiology laboratory information system.

\section{Authors' contributions}

SHB did the conception and design of the work, literature research, data collection, data analysis and interpretation, drafting of the article, critical revision of the article, and final approval of the article. RJ took part in the data collection, data analysis and interpretation, critical revision of the article, and final approval of the article. SR participated in the conception and design of the work, data analysis and interpretation, critical revision of the article, and final approval of the article. JPJW contributed in the data interpretation, critical revision of the article, and final approval of the article.
RJB have a hand in the data collection, critical revision of the article, and final approval of the article. NJMM helped in the data analysis and interpretation, critical revision of the article, and final approval of the article. PHJV cooperated in the conception and design of the work, data analysis and interpretation, drafting the article, critical revision of the article, and final approval of the article.

\section{Authors' information}

The authors work in the OLVG hospital that implemented SDD in the mid1980s. The SDD has been applied uninterruptedly. The authors have learned in these decades how to cope with abnormal carriership and how to eliminate multiresistant microorganisms. This unique situation is the setting in which the data should be interpreted.

\section{Funding}

This work was not funded in any way.

\section{Availability of data and materials}

Data are available in the electronic patient records. Anonymous datasets are generated and stored in a secured place in the hospital's electronic system for medical research.

\section{Ethics approval and consent to participate}

The local medical ethical review board (MEC OLVG) approved the study and waived informed consent due to its retrospective design in accordance with the Dutch and European legislation (study no. WO 13.063).

\section{Consent for publication}

Not needed as stated by the ethical review board.

\section{Competing interests}

The authors declare that they have no competing interests.

\section{Author details}

'Department of Intensive Care, OLVG Hospital, Oosterpark 9, 1091 AC Amsterdam, The Netherlands. ${ }^{2}$ TIAS School for Business and Society, Warandelaan 2, 5037 AB Tilburg, The Netherlands. ${ }^{3}$ Department of Medical Microbiology, OLVG Hospital, Oosterpark 9, 1091 AC Amsterdam, The Netherlands. ${ }^{4}$ Department of Intensive Care, Amphia Hospital, Molengracht 21, 4814 CK Breda, The Netherlands.

Received: 4 February 2019 Accepted: 19 May 2019

Published online: 07 June 2019

\section{References}

1. Silvestri L, van Saene HKF. Selective decontamination of the digestive tract: an update of the evidence. HSR Proc Intensive Care Cardiovasc Anesth. 2012:4:21-9.

2. Silvestri L, van Saene HKF, Milanese M, Gregori D, Gullo A. Selective decontamination of the digestive tract reduces bacterial bloodstream infection and mortality in critically ill patients. Systematic review of randomized, controlled trials. J Hosp Infect. 2007;65:187-203.

3. van Nieuwenhoven CA, Buskens E, Bergmans DC, van Tiel FH, Ramsay $\mathrm{G}$, Bonten MJM. Oral decontamination is cost-saving in the prevention of ventilator-associated pneumonia in intensive care units. Crit Care Med. 2004;32:126-30.

4. Liberati A, D'Amico R, Pifferi S, Torri V, Brazzi L, Parmelli E. Antibiotic prophylaxis to reduce respiratory tract infections and mortality in adults receiving intensive care. Cochrane Database Syst Rev. 2009;7:CD000022

5. Rogers CJ, van Saene HK, Suter PM, Horner R, Orme ML. Infection control in critically ill patients: effects of selective decontamination of the digestive tract. Am J Hosp Pharm. 1994;51:631-9.

6. Cuthbertson BH, Campbell MK, MacLennan G, et al. Clinical stakeholders' opinions on the use of selective decontamination of the digestive tract in critically ill patients in intensive care units: an international Delphi study. Crit Care. 2013;17:R266.

7. Silvestri $L$, van Saene HKF. Selective decontamination of the digestive tract does not increase resistance in critically ill patients: evidence from randomized controlled trials. Crit Care Med. 2006;34:2027-30.

8. Abecasis F, Sarginson RE, Kerr S, Taylor N, van Saene HKF. Is selective digestive decontamination useful in controlling aerobic gram-negative 
bacilli producing extended spectrum beta-lactamases. Microb Drug Resist. 2011;17:17-23

9. de Smet AM, Kluytmans JA, Blok HE, et al. Selective digestive tract decontamination and selective oropharyngeal decontamination and antibiotic resistance in patients in intensive-care units: an open-label, clustered group-randomised, crossover study. Lancet Infectious Dis. 2011;11:372-80

10. Hammond JM, Potgieter PD. Long-term effects of selective decontamination on antimicrobial resistance. Crit Care Med. 1995;23:637-45.

11. Plantiga NL, Bonten MJ. Selective decontamination and antibiotic resistance in ICUs. Crit Care. 2015;19:259.

12. Daneman N, Sarwar S, Fowler RA, Cuthbertson BH. Effect of selective decontamination on antimicrobial resistance in intensive care units: a systematic review and meta-analysis. Lancet Infect Dis. 2013;13:328-41.

13. Wayne $P(C L S I)$. Performance standards for antimicrobial susceptibility testing. Twentieth informational supplement. 2010.

14. European Committee on Antimicrobial Susceptibility Testing (EU-CAST). EUCAST breakpoint table version 1.0. http://www.eucast.org/clinical_ breakpoints/. Accessed 19 Aug 2018.

15. van der Voort PHJ, Buitinck S, Jansen RR, Franssen EJF, Determann RJ. Ten tips and tricks for successful digestive tract decontamination. Neth J Crit Care. 2019;27:87-90.

16. van der Voort PHJ, van Saene HKF. Selective digestive tract decontamination in intensive care medicine: a practical guide to controlling infection. Milan: Springer-Verlag Mailand; 2008

17. European centre for Disease Prevention and Control. European surveillance of healthcare-associated infections in intensive care units: HAl-Net ICU protocol, version 1.02. 2015.http://ecdc.europa.eu. Accessed 18 Jan 2019.

18. Beyersmann J, Gastmeier P, Schumacher M. Incidence in ICU populations: how to measure and report it? Intensive Care Med. 2014:40:871-6.

19. Houben AJM, Oostdijk EAN, van der Voort PHJ, Monen JCM, Bonten MJM, van der Bij AK. Selective decontamination of the oropharynx and the digestive tract, and antimicrobial resistance: a 4 year ecological study in 38 intensive care units in the Netherlands. J Antimicrob Chemother. 2014;69:797-804.

20. Wittekamp BH, Oostdijk EA, de Smet AMG, Bonten MJ. Colistin and tobramycin resistance during long- term use of selective decontamination strategies in the intensive care unit: a post hoc analysis. Crit Care. 2015;19:113.

21. Ochoa-Ardila ME, Garcia-Canas A, Gomez-Mediavilla K, et al. Long-term use of selective decontamination of the digestive tract does not increase antibiotic resistance: a 5-year prospective cohort study. Intensive Care Med. 2011;37:1458-65.

22. Heininger A, Meyer E, Schwab F, Marschal M, Unertl K, Krueger WA. Effects of long-term routine use of selective digestive decontamination on antimicrobial resistance. Intensive Care Med. 2006;32:1569-76.

23. Oostdijk EAN, de Smet AMGA, Blok HEM, et al. Ecological effects of selective decontamination on resistant gram-negative bacterial colonization. Am J Respir Crit Care Med. 2010;181:452-7.

24. Halaby $\mathrm{T}$, al Nalemi N, Kluytmans J, et al. Emergence of colistin resistance in Enterobacteriaceae after the introduction of selective digestive tract decontamination in an intensive care unit. AAC. 2013;57:3224-9.

25. Oostdijk EA, Kesecioglu J, Schultz MJ, et al. Effects of decontamination of the oropharynx and intestinal tract on antibiotic resistance in ICUs. A randomized clinical trial. JAMA. 2014:312:1429-37.

26. van der Bij AK, Frentz D, Bonten MJM. Gram-positive cocci in Dutch ICUs with and without selective decontamination of the oropharyngeal and digestive tract: a retrospective database analysis. J Antimicrob Chemother. 2016;71:816-20

27. Silvestri L, van Saene HKF, Casarin A, Berlot G, Gullo A. Impact of selective decontamination of the digestive tract on carriage and infection due to Gram-negative and Gram-positive bacteria: a systematic review of randomised controlled trials. Anaesth Intensive Care. 2008:36:324-38.

28. Wolfensberger A, Sax H, Weber R, Zbinden R, Kuster SP, Hombach M. Change of antibiotic susceptibility testing guidelines from CLSI to EUCAST: influence on cumulative hospital antibiograms. PLoS One. 2013;8:e79130

29. van der Bij AK, van Dijk K, Muilwijk J, et al. Clinical breakpoint changes and their impact on surveillance of antimicrobial resistance in Escherichia coli causing bacteraemia. Clin Microbiol Infect. 2012;18:E466-72.
30. Wittekamp BH, Plantinga NL, Cooper BS, et al. Decontamination strategies and bloodstream infections with antibiotic resistant microorganisms in ventilated patients. A randomized clinical trial. JAMA. 2018;320:2087-98.

\section{Publisher's Note}

Springer Nature remains neutral with regard to jurisdictional claims in published maps and institutional affiliations.
Ready to submit your research? Choose BMC and benefit from:

- fast, convenient online submission

- thorough peer review by experienced researchers in your field

- rapid publication on acceptance

- support for research data, including large and complex data types

- gold Open Access which fosters wider collaboration and increased citations

- maximum visibility for your research: over $100 \mathrm{M}$ website views per year

At $\mathrm{BMC}$, research is always in progress.

Learn more biomedcentral.com/submissions 